\title{
Diretrizes de Acessibilidade para Deficientes Visuais no Moodle: Guia para Professores
}

\author{
Edith dos Santos Lemos ${ }^{1}$, Giliane Bernardi ${ }^{1}$, Josiane Pozzatti Dal-Forno ${ }^{1}$ \\ ${ }^{1}$ Programa de Pós Graduação em Tecnologias Educacionais em Rede - PPGTER \\ Universidade Federal de Santa Maria - Av. Roraima 1000 - Santa Maria - RS - Brasil \\ edithdossantos@yahoo.com.br, \{giliane,josiane.dalforno\}@gmail.com
}

Resumo: A educação, que se pretende democrática, precisa atender a todos os educandos. Para apoiar este processo de inclusão, considerando a utilização de Ambientes Virtuais de Ensino e Aprendizagem (AVEA), diferentes diretrizes de acessibilidade vêm sendo criadas para orientarem a criação de cursos e recursos educacionais virtuais. No entanto, o que se observa é uma carência no que se refere a uma relação mais próxima entre as diretrizes e sua aplicabilidade em tais ambientes. Considerando o AVEA Moodle, este artigo tem como objetivo mostrar a criação de um guia de diretrizes de acessibilidade para apoiar os professores na elaboração de suas disciplinas ou cursos, quando estes necessitarem de auxilio para disponibilizarem materiais para alunos com deficiência visual e apresentá-las em um Objeto de Aprendizagem no Moodle. Após avaliação, foi possível concluir que este recurso pode contribuir para que os docentes tenham facilidade na organização e elaboração de conteúdos educacionais acessíveis.

Palavras-chave: Moodle, Acessibilidade, Objeto de Aprendizagem, Deficiência Visual.

\begin{abstract}
Education, which is to be democratic, must meet all learners. To support this process of inclusion, considering the use of Virtual Environments for Teaching and Learning (AVEA), different accessibility guidelines have been created to guide the creation of virtual courses and educational resources. However, what is observed is a shortage in relation to a closer relationship between the policies and their applicability in such an environment. Considering the AVEA Moodle, this article aims to show the creation of an accessibility guidelines guide to support teachers in developing their disciplines or courses when they require assistance to make available materials for visually impaired students and present them in a Learning Object in Moodle. After evaluation, it was concluded that this feature can help the teachers have ease in the organization and development of accessible educational content.
\end{abstract}

Keywords: Moodle Accessibility Learning Object, Visual Impairment.

\section{Introdução}

A inclusão educacional tem suas bases na legislação, contudo, para que realmente se efetive nos diferentes espaços de ensino, precisa superar muitos desafios. Nessa direção, o que temos visto é que com a tecnologia, o modo de se fazer inclusão mudou.

A revolução tecnológica tem modelado novos espaços e tempos e estruturado novos conceitos culturais e sociais. As Tecnologias da Informação e da Comunicação (TIC) começam a potencializar a construção de uma sociedade que, ao renunciar às lógicas de exclusão, aproxima-se da utopia possível de inserção para todos os seus atores sociais (CONFORTO e SANTAROSA, 2002). 
A revolução a qual as autoras se referem reforça a grande potencialidade que as tecnologias possuem para que se criem novas maneiras e novas formas de aplicá-las a favor da inclusão em todos os meios, inclusive o da educação. Segundo Mozzaquatro e Medina (2008), no atual cenário é fundamental que a educação integre-se à sociedade do conhecimento. Uma das formas de realizar essa tarefa é desenvolver estratégias de aprendizagem, mediadas por tecnologia da informação, um exemplo são os Ambientes Virtuais de Ensino e Aprendizagem (AVEA). Estes ambientes encontram-se em grande expansão de acordo com Sabattini (2007), sendo que um dos que merece destaque é o AVEA Moodle. Em contrapartida, para Silva et al. (2013), as interfaces responsáveis pela exibição do conteúdo em tais ambientes, em sua maioria, não seguem diretrizes para acessibilidade devido a forma incorreta de como os conteúdos são disponibilizados aos estudantes.

Considerando o que foi exposto, foi elaborado um conjunto de diretrizes de acessibilidade para criação de cursos em AVEAS, focando, neste caso, no ambiente virtual Moodle. Objetivou -se, com este conjunto de diretrizes, possibilitar ao professor a elaboração de conteúdos mais acessíveis no Moodle, voltado para estudantes com deficiência visual. Para a apresentação deste conjunto de diretrizes aos professores, agregando a elas um detalhamento teórico, bem como sugestões de como implantar as mesmas no Moodle, foi desenvolvido um Objeto de Aprendizagem do tipo Livro (recurso educacional presente no Moodle), para ser disponibilizado aos professores sempre que eles desejarem utilizar tais diretrizes na criação de uma disciplina. Este artigo tem como objetivo apresentar as diretrizes e o Objeto de Aprendizagem desenvolvido.

\section{Deficiência Visual e Educação}

A educação das pessoas com deficiências no Brasil é um tema bastante discutido por vários autores, tais como Dal Forno (2005), Nuernberg (2008), Pletsch (2014) e Taveira e Rosado (2010) destacam e (Dallabona, 2011, p.6) afirma que as diferenças não devem ser apenas aceitas como também acolhidas pelos professores, adotando, assim, práticas criativas, adaptando o currículo pedagógico, revendo posturas e construindo uma nova filosofia educativa. O desafio é estender o princípio da inclusão à maioria dos acadêmicos e ter em mente que o principal propósito é facilitar a aprendizagem.

Considerando a deficiência visual, foco deste trabalho, para Brumer et al. (2004), a falta ou redução de visão não é o principal obstáculo para a inclusão de pessoas com deficiência visual como cidadãos, plenos de direitos e deveres. Caso lhes sejam oferecidas as condições de aprendizado e os meios de desenvolver e aplicar suas habilidades, tais pessoas têm condições de andar sozinhos, estudar, trabalhar e de participar da vida social, econômica, cultural e política da sociedade.

O processo de ensino e aprendizagem passa a ter significado quando o professor dá condições necessárias para que o acadêmico participe ativamente das atividades, contribuindo significativamente para o processo de construção social desse. A realização da leitura do material somada à descrição das imagens, quadros e tabelas fazem uma grande diferença no que se refere ao acesso à informação, porém, se o professor-tutor não tem o devido preparo, pode influir negativamente no entendimento do acadêmico (CARVALHO e DALTRINI, 2011).

Para Dallabona (2011, p.8), "é preciso ensinar propiciando condições, criando situações, oferecendo oportunidades ao acadêmico para se desenvolver e ampliar suas capacidades de produzir o conhecimento". No caso de acadêmicos cegos ou com baixa 
Ainda de acordo com a autora, as TIC ampliam substancialmente o universo dos acadêmicos com deficiência, sendo que, desde que bem direcionadas e aplicadas, elas podem facilitar seus relacionamentos interpessoais e até servir de estímulo, pois alguns desses acadêmicos são desmotivados frequentemente pela sociedade, que confunde deficiência com ineficiência. A próxima seção destaca o papel das tecnologias como apoio a acessibilidade.

\section{Acessibilidade e Tecnologias}

O avanço do movimento de inclusão de pessoas com deficiência nos contextos educacionais passou a exigir ações relacionadas ao atendimento às diversidades dos processos de aprendizagem, em um desafio constante às formas tradicionais de organização do trabalho pedagógico. Novas formas da ação pedagógica, orientadas para a diversidade dos processos do aprender, são necessárias para que possamos falar de inclusão, do contrário, continuaremos repetindo situações em que o sucesso da experiência educacional decorria somente da adaptação dos sujeitos ao processo, como no modelo educacional da integração (CARVALHO, 2004).

Para auxiliar no processo da inclusão digital de pessoas com deficiência visual, existem as tecnologias assistivas, como alguns softwares leitores de tela. Tecnologia assistiva é utilizada para identificar todo o arsenal de recursos e serviços que contribuem para proporcionar ou ampliar habilidades funcionais de pessoas com deficiência e consequentemente promover vida independente e inclusão (BERSCH E TONOLLI, 2010). Para Silveira e Batista (2011), pedagogias inovadoras e tecnologias assistivas têm, assim, um vínculo natural, dado pelo foco nas pessoas e na sua autonomia. $\mathrm{O}$ acesso à informação e a meios e recursos de comunicação e expressão são essenciais para a inclusão social e para a aprendizagem.

Considerando um AVEA, o aluno tem acesso aos recursos educacionais e atividades disponíveis por meio de um leitor de tela, ou seja, da mesma forma que ele toca um objeto palpável, ele deve ouvir detalhadamente a explicação no ambiente virtual, para que ele possa se situar e compreender. No entanto, os leitores de tela, por si só, não atendem às necessidades dos estudantes. Se os materiais educacionais não tiverem sido inseridos no ambiente levando em consideração alguns critérios específicos de acessibilidade, eles não conseguirão fornecer as informações pertinentes aos estudantes.

Segundo Coelho (2011), o Moodle atende minimamente aos requisitos de acessibilidade previstos pelas normas da W3C (World WideWeb Consortium) em todas as suas versões e não requer nenhum desenvolvimento de novos componentes para que isto seja possível. É necessário apenas que se adotem algumas boas práticas de desenvolvimento web ao se configurar e criar o ambiente do curso ou disciplina. A próxima seção destaca algumas propostas e modelos que sugerem diretrizes de acessibilidade para deficientes visuais e que podem ser implantadas em AVEA como o Moodle.

\section{Trabalhos Correlatos}

Conforme descrito na introdução, esta pesquisa teve como objetivo central o desenvolvimento de um conjunto de diretrizes de acessibilidade para o AVEA Moodle, com foco na deficiência visual. Para elencar tais diretrizes, diferentes trabalhos de outros autores, bem como modelos e diretrizes universais, foram considerados. Esta 
seção tem como objetivo apresentar alguns destes trabalhos, apontando as contribuições dos mesmos e como foram utilizados tais trabalhos na presente proposta. Entre os trabalhos analisados, podem ser destacados os apresentados por Fialho (2014) e Silva etal. (2013).

Segundo Silva et al. (2013) em sua pesquisa, vários aspectos foram avaliados na construção de diretrizes para melhorar a acessibilidade no Moodle. Buscou o embasamento teórico do Desenho Universal para a Aprendizagem e com isso a organização acessível do ambiente. O trabalho apresenta uma análise sobre a acessibilidade dos conteúdos disponibilizados no Moodle, em duas disciplinas do Curso de Pedagogia a Distância, do Centro de Educação a Distância (CEAD) da Universidade do Estado de Santa Catarina (UESC) e Universidade Aberta do Brasil (UAB). Fialho (2014) em sua pesquisa desenvolveu um estudo de caso em uma universidade federal para investigar o que os docentes conheciam sobre acessibilidade e colocavam em prática na publicação de materiais educacionais no ambiente Moodle. O trabalho de Fialho (2014) teve como objetivo promover a acessibilidade do ambiente de aprendizagem virtual da instituição, orientando docentes na publicação de materiais e técnicos de informática na manutenção do ambiente Moodle. Com base nas experiências descritas pelo corpo docente e na avaliação do Moodle, foram propostas recomendações para os docentes e para a equipe responsável pela manutenção do Moodle.

Com relação a normas e modelos utilizados nesta pesquisa, tomou-se como base os princípios do Desenho Universal que, de acordo com Cast (2011) é a concepção de produtos, ambientes, programas e serviços a serem usados, na maior medida possível, por todas as pessoas, sem necessidade de adaptação ou projeto específico. Ainda, foram consideradas as diretrizes de acessibilidade do Modelo de Acessibilidade em Governo Eletrônico e-Mag (BRASIL, 2011), que é a referência nacional para desenvolvimento de sites públicos que contemplem as questões de acessibilidade.

Todos os trabalhos e modelos analisados buscaram apresentar um conjunto semelhante de diretrizes que podem ser utilizados de forma a criar ambientes acessíveis. No entanto, todos disponibilizam tais diretrizes sem um auxílio mais detalhado de como implantar as mesmas em AVEA, sendo que mesmo os trabalhos que tomaram o Moodle como exemplo não detalharam como aplicar tais diretrizes. Ainda, estas informações encontram-se disponibilizadas por meio de documentos estáticos e, em sua maioria, descritas de maneira puramente textual.

Considerando estas questões, surgiu a proposta de construção de um Objeto de Aprendizagem do tipo Livro (recurso presente no Moodle), que pudesse apresentar tais diretrizes de forma mais interativa, por meio de links, imagens, textos, entre outras mídias, integrado ao ambiente Moodle, permitindo a ligação dos conceitos descritos diretamente com suas funcionalidades no ambiente. A próxima seção apresenta este desenvolvimento.

\section{Objeto de Aprendizagem para Criação de Cursos Acessíveis no Moodle}

Para Viera e Nicoleit (2007), pode-se definir um Objeto de Aprendizagem (OA) como um recurso digital que pode ser utilizado como auxílio ao processo de ensinoaprendizagem e que tem a capacidade de ser reutilizado em vários contextos de maneira a facilitar a apropriação do conhecimento. Considerando os recursos educacionais presentes no Moodle, classifica-se o Objeto de Aprendizagem desenvolvido neste trabalho como um recurso do tipo Livro, sendo que o mesmo foi desenvolvido de acordo com as funcionalidades e especificidades deste recurso. O Livro é uma atividade 
formada por um conjunto de páginas. No Moodle, é um material didático digital com conteúdo para estudo (TUTORIAL MOODLE, 2014).

As funcionalidades que foram atendidas com este OA podem ser destacadas:

- Disponibilização aos professores das diretrizes desenvolvidas de uma forma mais eficiente, pelo fato do livro ser um recurso educacional do ambiente Moodle (embutido no ambiente), em vez de ser um guia separado;

- Apresentação das informações de uma forma mais organizada e funcional, proporcionando a inserção de links para cada diretriz, que permitem que o professor possa se encaminhar diretamente para a diretriz que deseja, tornando o acesso mais direto e otimizado para as funcionalidades desejadas;

- Exemplos de como implementar as diretrizes no Moodle, incorporando links que levam o professor diretamente para a funcionalidade que está sendo explicada, possibilitando ao mesmo executar as instruções de forma mais facilitada. Por exemplo, uma das diretrizes orienta o professor com relação a inserção de textos explicativos para as imagens inseridas no ambiente. Junto a descrição e explicação da diretriz, são exibidas figuras ilustrativas que mostram ao professor esta funcionalidade no Moodle, bem como são apresentados links ao professor que conduzem diretamente para o processo de inserção de uma figura com legenda explicativa;

- Disponibilização de exercícios que podem ser realizados dentro do próprio ambiente (atividades de treinamento), relacionadas a cada diretriz de acessibilidade e sua implantação no ambiente. Com isto, o professor pode acessar as informações uma determinada diretriz, e realizar exercícios que o orientem para a implementação da mesma no Moodle;

- Inserção de links que conduzem às referências bibliográficas que foram utilizadas como base para o desenvolvimento das diretrizes, possibilitando ao professor poder acessar os documentos (na íntegra) que ajudaram a compor as diretrizes. No caso deste trabalho, estão sendo disponibilizados os artigos, modelos e normas que foram apresentados na seção de trabalhos correlatos, bem como artigos e documentos que trazem informações gerais sobre o tema acessibilidade e deficiência visual;

- Disponibilização de um glossário (recurso educacional presente no Moodle), em substituição a textos explicativos que estariam presentes no meio do texto, para termos técnicos e explicações adicionais. O Glossário funciona por meio da associação de links nestas expressões ou termos, conduzindo a uma explicação apenas se o usuário clicar nos mesmos. Isto torna a leitura mais fluída e direta para os usuários que não quiserem esta explicação, bem como torna a compreensão mais completa para os que desejarem saber mais sobre determinado assunto.

Finalmente, como contribuição geral, pode-se afirmar que a criação do guia por meio de um livro do Moodle, pode tornar a leitura e implementação das diretrizes um processo mais atrativo e motivacional para o professor, considerando a diversidade de formas (áudios, vídeos, textos, links, etc) implementadas pelo mesmo, bem como o a riqueza e detalhamento na apresentação do conteúdo das diretrizes ao professor. As próximas subseções destacam os procedimentos metodológicos e o desenvolvimento do mesmo. 


\subsection{Aspectos Metodológicos}

O processo de desenvolvimento do Objeto de Aprendizagem teve como base a abordagem de Design Instrucional, a qual pode ser definida como o processo de identificar um problema de aprendizagem e desenhar, desenvolver, implementar e avaliar uma solução para este problema (FILATRO, 2008). Entre os modelos mais conhecidos de Design Instrucional, destaca-se o modelo ADDIE, formado pelas etapas de Análise (Analisys), Desenho (Design), Desenvolvimento (Development), Implementação (Implementation) e Avaliação (Evaluation).

Na primeira etapa, de Análise, foi definido o público-alvo do OA, que foram os professores que usaram o objeto para guiá-los na elaboração e disponibilização do material. Ainda, foram definidos o tema e o escopo, sendo que podemos considerar Acessibilidade para Deficientes Visuais no Moodle como o tema central, e como escopo, os conteúdos associados às diretrizes de acessibilidade desenvolvidas. As diretrizes foram desenvolvidas com base nos trabalhos analisados e já descritos.Na fase de Desenho, são planejadas as atividades e unidades de aprendizagem do OA. Considerando a organização do livro criado, o mesmo é composto pelas diretrizes de acessibilidade, sendo que cada página do livro é composta por uma diretriz e a explicação de como ela deve ser implementada (como se fossem capítulos do livro). Ainda, para cada diretriz apresentada, são propostas atividades, links para assuntos relacionados e indicação de sua implementação no Moodle.

No que concerne a fase de Desenvolvimento, cabe salientar que a primeira versão do livro foi desenvolvida como um protótipo inicial, que foi utilizado e avaliado por professores, sendo que, mediante esta primeira avaliação, o mesmo foi refinado, passando pelo processo detalhado de desenvolvimento para sua versão final. Considerando este protótipo foram desenvolvidas as etapas de Implementação e Avaliação. Para a avaliação, três professores foram convidados para implementaremuma disciplina fictícia dentro do ambiente Moodle, sendo que os mesmos tiveram acesso ao Livro de diretrizes junto ao ambiente do curso. A partir do desenvolvimento deste ambiente e utilização do Livro de Diretrizes de Acessibilidade, os mesmos avaliaram o Objeto de Aprendizagem considerando aspectos relacionados à Usabilidade, Experiência de Uso e Funcionalidade.

Nesta primeira versão também foi criado um curso teste, pelos autores desta pesquisa, onde foram utilizadas todas as diretrizes, sendo que este curso foi realizado por dois alunos deficientes visuais, com o objetivo de avaliar as diretrizes sob o ponto de vista dos estudantes. As próximas seções destacam as diretrizes e OA criado, bem como a avaliação realizada.

\subsection{Diretrizes de acessibilidade para desenvolvimento de cursos no Moodle}

As diretrizes foram criadas e implementadas em quatro grupos: diretrizes para configuração do ambiente, diretrizes para definição e organização dos recursos, diretrizes para definição e organização das atividades e recomendações gerais. Cabe salientar que as diretrizes são de caráter genérico e foram propostas baseadas na versão 2.7.4+ do Moodle.

As diretrizes para configuração do ambiente estão divididas em:

- Habilitar o leitor de tela (FIALHO, 2014);

- Configurar blocos e tópicos (DALCIN, 2015);

- Oferecer contraste mínimo entre plano de fundo e primeiro plano (BRASIL, 2011); 
- Descrever todas as figuras (SILVA et al, 2013; BRASIL, 2011);

O Conjunto de diretrizes referente à definição e organização dos recursos, estão divididas em:

- Sugerir adequadamente o conteúdo dos Links (BRASIL, 2011);

- Disponibilizar os vídeos acompanhados de descrição sonora e textual(BRASIL,2011);

- Colocar as informações em diferentes mídias (FIALHO, 2014);

O Conjunto de diretrizes para definição e organizações das atividades estão divididas em:

- Permitir o uso de diferentes formas de linguagem (CAST, 2011; SILVA et al, 2013);

- Elaborar orientações claras e objetivas para as atividades (LEMOS, 2015);

- Atribuir editor de texto simples para Wiki (LEMOS, 2015; DALCIN, 2015);

- Organizar os estudantes em grupos pequenos para o chat (LEMOS, 2015);

Priorizar as tarefas de arquivo único (SILVA et al, 2013).

Além da identificação de diretrizes específicas, voltadas para melhor utilização do AVEA Moodle, algumas diretrizes com recomendações gerais também foram elencadas, com o objetivo de auxiliar os professores na elaboração dos conteúdos educacionais destacando sugestões de como construir textos mais acessíveis e recomendações para descrição de abreviações, siglas, termos técnicos ou especializados.

\subsubsection{Apresentação do Objeto de Aprendizagem Livro}

O livro desenvolvido é composto pelas diretrizes de acessibilidade descritas na seção anterior, sendo que cada diretriz é apresentada em um capítulo (uma página) contendo uma descrição da mesma. Cada página funciona como uma espécie de tutorial para que o docente que está lendo não fique com dúvidas, contendo informações adicionais sempre que necessário. Ainda, associado a cada diretriz encontra -se um link para um convite de desafio (exercício). Nesta página ainda encontra-se disponível conteúdos de apoio.

O livro possui uma página inicial, sendo que, a partir desta, pode-se navegar sequencialmente, ou por meio do sumário que fica no lado esquerdo da tela, no qual têm-se os capítulos, por meio dos quais pode-se acessar diretamente a diretriz desejada. Este é o formato padrão do Moodle para configuração de recursos do tipo Livro.

Sua organização ocorre da seguinte forma: introdução, um capítulo para cada grupo de diretrizes já descritas, conclusão, glossário e referências. A figura 1 exibe um exemplo de página com conteúdo sobre uma diretriz, neste caso, a diretriz de número 3.2, que descreve sobre a importância de ser disponibilizada uma alternativa sonora ou textual a vídeos que não contenham áudio. Após a conclusão, passou-se a avaliação do protótipo inicial, destacado na próxima seção. 


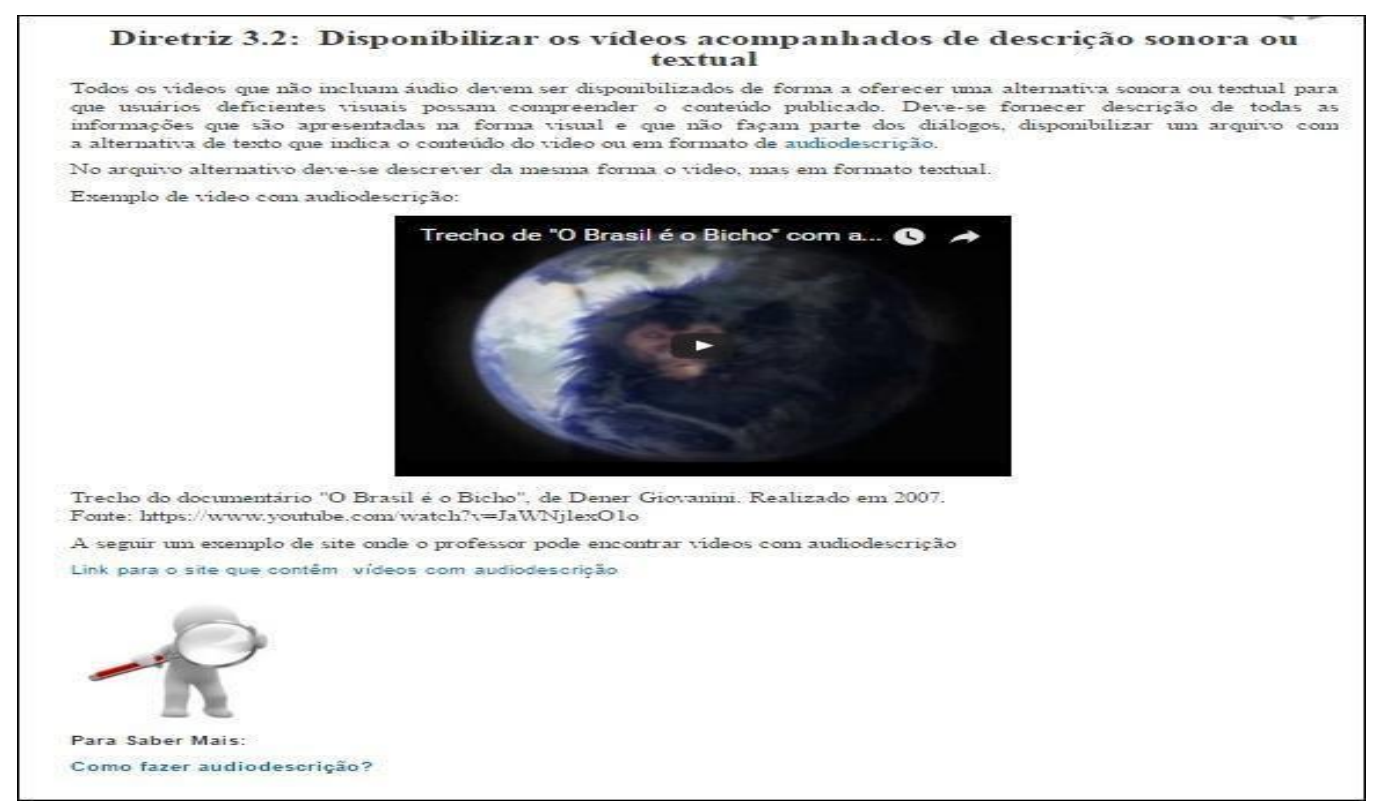

Figura 1 - Exemplo de uma página de exibição de diretriz no Livro

\subsection{Resultados Parciais e Modificações}

Conforme já descrito na seção 5.1, a avaliação foi realizada por 3 (três) professores e 2 (dois) estudantes cegos, com o objetivo de avaliar a funcionalidade do objeto criado, bem como a efetividade das diretrizes elencadas. Os professores desenvolveram um curso exemplo tomando como referencia as diretrizes e, após, responderam a um questionário que envolvia questões sobre a elaboração das diretrizes, formatação do livro. Conforme as respostas fornecidas, tanto as diretrizes como o objeto tiveram um percentual de aprovação de $90 \%$, destacando que $82 \%$ concordaram que elas ajudarão os professores na elaboração de seus cursos mais acessíveis, principalmente no que tange ao acesso do aluno deficiente visual. Com relação aos estudantes, estes realizaram o curso teste e, após, também foram convidados a tecer suas impressões sobre o mesmo com relação à critérios de acessibilidade. Com base em seus comentários foi possível observar se as diretrizes conseguiram atender a todas as necessidades de criação de um curso acessível ou se modificações e acréscimos seriam necessários.

Como resultados dessa avaliação, foram identificadas algumas modificações a serem realizadas, tanto em relação ao conteúdo das diretrizes como em relação a organização e exibição das mesmas no livro. Inicialmente, a diretriz 'Priorizar as tarefas de arquivo único", foi alterada passando a sugerir o envio de tarefas no formato de texto online. Esta alteração ocorreu devido a não conseguirem enviar o arquivo. O relato do aluno A demonstra este problema quando afirma que: "o fato de ter que entrar em outra página pra enviar o arquivo, e ter que clicar em vários links pra tentar chegar ao botão 'Procurar' não agrada muito o usuário, e por mais que eu tenha tentado enviar o arquivo, depois que cliquei em enviar arquivo, não achei o botão procurar" Ainda, o aluno B expõe que: "o caminho para postar a tarefa poderia ser menor e o layout da página tem que mudar pois não consigo chegar até o botão para procurar o arquivo $e$ postá-lo". Assim,por meio das avaliações foi possível constatar que mesmo esta opção torna-se complexa, devido a abrir várias telas. Cabe salientar que Silva et al. (2013) sugere envio de arquivos únicos como sendo uma atividade acessível, o que não se mostrou uma proposta viável neste projeto. 
Outra alteração feita no livro foi com relação aos links "Para saber mais", que estavam junto aos desafios. Eles passaram para o final da página de cada diretriz. Como descrito anteriormente, o professor é convidado a realizar um exercício/desafio, mas não é elemento obrigatório na leitura do livro, que não segue uma organização linear. Nestecaso, se o professor não acessasse a página de desafios, não teria acesso direto aos links com conteúdos adicionais. Considerando que a página que contém a diretriz em si é o elemento central, a inserção destes links junto à mesma, torna mais visível este conteúdo. Em função da avaliação das diretrizes o convite para o desafio também mudou. Elaborou-se uma chamada tentando atrair e instigar o professor a realizá-lo, pois observou-se que poucos professores acessaram esta página. Por fim, com relação à diretriz "Oferecer contraste mínimo entre plano de fundo e primeiro plano", foi acrescentado um trecho para complementar esta diretriz, disponibilizando um software que calcula o contraste embutido na página do livro.

\section{Considerações Finais}

Este artigo teve como objetivo apresentar as diretrizes e o desenvolvimento de um Objeto de Aprendizagem para apoiar a criação de cursos acessíveis dentro do AVEA Moodle. O OA em questão foi desenvolvido como um recurso do tipo Livro, ferramenta disponível na plataforma Moodle. Considera-se que o meio escolhido pode ser considerado atrativo e motivador aos professores, auxiliando-os de forma mais interativa e funcional a desenvolverem seus cursos no Moodle. Espera-se que esta pesquisa venha contribuir com os docentes que tem alunos com deficiência visual, facilitando a organização e elaboração dos conteúdos e também os alunos deficientes visuais que terão maior produtividade, pois poderão aprender e participar de forma igualitária.

Como perspectivas de trabalhos futuros, pretende-se disponibilizar a versão revisada novamente para avaliação junto a professores e estudantes com deficiência visual, para que, futuramente, o mesmo possa ser disponibilizado para toda a comunidade acadêmica.

\section{Referências Bibliográficas}

BERSCH, R; TONOLLI, J.C. Tecnologia Assistiva, 2010. Disponível em: <www.assistiva.com.br.>. Acesso em: setembro 2014.

BRASIL. Ministério de Planejamento, Orçamento e Gestão. Secretaria de Logística e Tecnologia da Informação. e-MAG - Modelo de Acessibilidade de GovernoEletrônico. Secretaria de Logística e Tecnologia da Informação, 2011.

BRUMER, A; PAVEI,K; MOCELIN, G. D. Saindo da "escuridão": perspectivas dainclusão social, econômica, cultural e política dos portadores de deficiência visual em Porto Alegre. Sociologias, Porto Alegre, ano 6, n 11, jan/jun 2004, p. 300327.

CARVALHO ,J. O. F..; DALTRINI, B.M. Educação a Distância: uma forma deInclusão do Deficiente Visual no Ensino Superior.2011.

CARVALHO, R. E. Educação Inclusiva com os pingos nos "is".Porto Alegre: Mediação, 2004.

CAST. Design Universal para Aprendizagem. GuidelinesVersion 2.0. Wakefield, 2011. 
COELHO, C. M. Acessibilidade para pessoas com deficiência visual no Moodle. Linhas Críticas, Brasília, DF, v. 17, n. 33, p. 327-348, maio/ago.2011.

CONFORTO. D; SANTAROSA. L. M. C. Acessibilidade à Web: Internet para Todos.RENOTE - Revista Novas tecnologias na Educação.Vol. 5, n1, 2007.

DALCIN, E. Interfaces Acessíveis no Moodle baseadas no padrão WCAG 2.0 para alunos cegos. Dissertação (Mestrado em Tecnologias Educacionais em Redes). Universidade Federal de Santa Maria, Santa Maria, 2015.

DAllabONA, G. K. (2011, 06). A Inclusão de Deficientes Visuais no Curso Superior na Educação a Distância.

DAL FORNO, J, P. Imaginários e saberes docentes na escola inclusiva: um estudo dos processos de formação e autoformação. Dissertação (Mestrado em Educação). Universidade Federal de Santa Maria, Santa Maria, 2005.

LEMOS, E, S. Acessibilidade para pessoas com deficiência visual em cursos no Moodle: Guia para professores.Dissertação (Mestrado em Tecnologias Educacionais em Redes). Universidade Federal de Santa Maria, Santa Maria, 2015.

FIALHO, L, G. Acessibilidade Web em Ambiente Virtual de Aprendizagem: umestudo de caso na UNIPAMPA, Alegrete, Universidade Federal do Pampa, 2014.

FILATRO, A. Design na Prática. São Paulo: Person Education do Brasil,2008.

MOZZAQUATRO. P. M.; MEDINA, R. D. Avaliação do Ambiente Virtual de Aprendizagem Moodle sob diferentes visões: aspectos a considerar. RENOTE RevistaNovas tecnologias na Educação, v. 6, n. 2, dez. 2008.

PLETSCH. M. D.Educação Especial e Inclusão Escolar: Políticas, Práticas Curriculares e Processos de Ensino e Aprendizagem.Revista Poíesis Pedagógica - Revista do PPGEDUC -Universidade Federal de Goiás - Campus Catalão, GO.

SABBATINI,R.M.E . Ambiente de Ensino e Aprendizagem via Internet.APlataforma Moodle. 2007.

SILVA. S. C.; BOCK.K. L; BECHE. E. R. C.; GOEDERT, L. Ambiente Virtual de Aprendizagem Moodle: Acessibilidade nos Processos de Aprendizagem na Educação a Distância/CEAD/UDESC.In: X Congresso Brasileiro de Ensino Superiora Distância ,Belém/PA, 2013.

SILVEIRA, C.; BATISTA.M.H.E. Análise de Softwares Leitores de Tela noAmbiente Virtual de Aprendizagem Moodle: Um estudo através de requisitos de softwares.iTEC, v.2,n2,jul.2011.

TAVEIRA, C.C.; ROSADO, S, A. Tecnologia Assistiva(TA) e alunos com Deficiência Visual: Um recorte sobre representações na Disputa entre DOSVOX e BRAILLE.RENOTE - Revista Novas tecnologias na Educação. Vol. 8 Num.2, 2010.

TUTORIAL MOODLE. UNIVERSIDADE FEDERAL DE SANTA MARIA, Núcleo de Tecnologia Educacional, Tutorial Moodle, 2014.

VIEIRA, M. C. E.; NICOLEIT. R.E. Desenvolvimento de Objeto de Aprendizagem, baseado em Especificações de Normatização SCORM, para o Caso de Suporte à Aprendizagem de Funções. RENOTE - Revista Novas tecnologias na Educação, VOL. 5, N.1. 2007. 